\title{
Do folic acid supplements reduce facial clefts?
}

\author{
Is folic acid deficiency associated with facial clefts?
}

\author{
Wilcox AJ, Lie RT, Solvoll K, et al. \\ Folic acid supplements and risk of facial clefts: national population \\ based case-control study. Br Med J 2007; 334(7591):433-434
}

Design This was a national population-based case-control study in Norway.

Case and control selection Cases consisted of all newborn infants born from 1996-2001 who had been referred for surgical treatment of a cleft [either cleft lip with or without cleft palate (377 children) or cleft palate only (196 children)]. Controls (763) were a randomly selected at an average of four per 1000 live births (identified through the medical birth registry of Norway).

Data analysis Data were collected using questionnaires. Crude odds ratios (OR) were calculated and adjustments for potential confounders (diet and multivitamins, mother's education, mother's employment during early pregnancy, smoking, alcohol consumption, and year of baby's birth) were made.

Results Folic acid supplementation during early pregnancy $(400 \mu \mathrm{g} /$ day) was associated with a reduced risk of isolated cleft lip with or without cleft palate after adjustment for multivitamins, smoking, and other potential confounding factors [adjusted OR, 0.61; 95\% confidence interval $(\mathrm{Cl}), 0.39-0.96]$. Independent of supplements, diets rich in fruits, vegetables and other high-folate-containing foods reduced the risk somewhat (adjusted OR, $0.75 ; 95 \% \mathrm{Cl}, 0.50-1.11$ ). The lowest risk of cleft lip was among women who ate folate-rich diets who also took folic acid supplements and multivitamins (adjusted OR, 0.36; $95 \% \mathrm{Cl}$, $0.17-0.77$ ). Folic acid provided no protection against cleft palate alone (adjusted OR, 1.07; 95\% Cl, 0.56-2.03).

Conclusions Folic acid supplements during early pregnancy seem to reduce the risk of isolated cleft lip (with or without cleft palate) by about a third. Other vitamins and dietary factors may provide additional benefit.
Address for correspondence: AJ Wilcox, Epidemiology Branch, National Institute of Environmental Health Sciences, National Institutes of Health, Durham NC 27709, USA.E-mail: wilcox@niehs.nih.gov

\section{Commentary}

Facial cleft, along with spina bifida and anencephaly, is among the group of abnormalities known as neural tube defects. A recent Cochrane review ${ }^{1}$ concluded that, "periconceptional folate supplements have a strong protective effect against neural tube defects" and that, "information about folate should be more widely available throughout the health and education systems." Indeed, since 1998, flour has been fortified with folic acid in both the US and Canada. Chile adopted this measure in 2000 but as yet, no European country has done so.

This large Norwegian study collected data from two groups of patients. Questionnaires were sent to the mothers whose babies were born between 1996 and 2001 and had been referred for surgical treatment of a cleft lip and/ or palate. The control group was randomly selected babies who did not have cleft lip from the medical births registry in Norway, and 763 mothers of these babies agreed to participate and were also sent questionnaires.

As well as collecting demographic data and information on smoking, alcohol, drugs and other exposures during pregnancy, a nutritional questionnaire was filled out. Mothers were asked if they had taken folic acid supplements and, if so, the specific product name. They were also asked during which months of their pregnancy the supplements were taken and participants were invited to send in empty pill bottles or labels for confirmation. Thus, folic acid dose was calculated for $81 \%$ of participants and, for the remainder, dose calculation was carried out according to the year the supplement was taken, as Norwegian dose policy changed in 1998.

It was recognised by the researchers that the reconstruction of mothers' folic acid exposure history was retrospective, and there may have been problems with reporting accuracy and recall bias. In addition, the fact that some mothers were taking multivitamins around the time of conception also needed to be taken into account.

Results showed that once the OR were adjusted for dietary folate intake as well as multivitamin use, the groups of women taking $400 \mu \mathrm{g}$ or more per day of folic acid supplements were at the lowest risk of giving birth to a baby who had a cleft. This level of folic acid supplementation reduces the risk of isolated cleft lip (with or without cleft palate) by a third, but seemed to have no effect on the risk of cleft palate alone. This is consistent with embryological and epidemiological data showing distinct causal mechanisms for these two types of defects. Thus, this study further strengthens the evidence that folic acid supplementation helps reduce certain embryological defects, and this includes cleft of the lip with or without cleft of the palate.

In order to be effective, folic acid needs to be taken in early pregnancy, preferably from around the time of conception: lip fusion takes place during weeks 5 and 6 of life, and palatal fusion occurs during weeks 7-10. Many women are only just realising that they may be pregnant at that time, and it may be too late to take advantage of folic acid supplementation. 
A UK survey carried out in 2003 found that $87 \%$ of women taking part understood the benefits of supplementing their diet with folic acid, but just over $50 \%$ were doing so. The same survey showed that $40 \%$ of women started taking folic acid later than 6 weeks after conception, and only $20 \%$ of women were taking the supplement when planning their pregnancy.

One approach to this problem was piloted in The Netherlands and targeted women returning to pharmacies for their oral contraceptives, with information being provided about the benefits of folate. A further initiative carried out in The Netherlands in 1995 was a national mass media campaign to inform patients and professionals about folic acid supplements. Following this campaign, a slight reduction in the prevalence of neural defects was noted. This approach does not target individual women and a followup study in 1998 concluded that although awareness and use of folic acid had increased, socioeconomic differences remained and even increased. Other studies have also shown that knowledge about the protective effect of folic acid varies between socio-economic groups, with women in lower groups demonstrating reduced awareness.

The US and Canada have recognised for 10 years that the only effective method of ensuring that all women receive folic acid supplements from around the time of conception is to provide supplements in food. In May 2007, the UK Food Standards Agency Board agreed unanimously that mandatory fortification of folic acid should be introduced. This board is awaiting a review from the Scientific Advisory Committee in Nutrition to be completed at the end of 2008 .

Dental practitioners will draw a parallel here with the campaign to fluoridate water supplies. As with the fluoridation issue, concerns about compulsory folic acid fortification have been raised. It is also recognised that most people who will eat fortified food will not be potential pregnant mothers. There have been some concerns that folic acid supplements may mask vitamin B12 deficiency in the elderly, and it is further claimed that folic acid may also enhance tumour growth.

As with the fluoride debate, these concerns must be investigated even though some may turn out to be spurious. Nevertheless, the evidence for the beneficial effects of folate supplements in reducing the incidence of cleft lip with or without palate is further strengthened by this paper.

\section{Julian O'Neill}

Orthodontic Department, Kettering General Hospital NHS Trust, Kettering, Northamptonshire, UK

1. Lumley J, Watson L, Watson M, Bower C. Periconceptional supplementation with folate and/or multivitamins for preventing neural tube defects. Cochrane Database Syst Rev 2001; issue 3.

Evidence-Based Dentistry (2008) 9, 82-83. doi:10.1038/sj.ebd.6400600 